O-04-1102

血液

\title{
Serum levels of free light chain before and after chemotherapy in primary systemic AL amyloidosis
}

Masayuki Matsuda, Toshiyuki Yamada**, Takahisa Gono, Yasuhiro Shimojima, Wataru Ishii, Tomohisa Fushimi, Kazuo Sakashita*, Kenichi Koike* and Shu-ichi Ikeda

From the Third Department of Medicine and *the Department of Pediatrics, Shinshu University School of Medicine, Matsumoto and **the Department of Clinical Pathology, Juntendo University School of Medicine, Tokyo

Received for publication:

Accepted for publication:

Reprint: Reprint requests should be addressed to Dr. Masayuki Matsuda, the Third Department of Medicine, Shinshu University School of Medicine, 3-1-1 Asahi, Matsumoto, 390-8621

Running title: FLC in AL amyloidosis

連絡先

住所長野県松本市旭3-1-1 信州大学医学部第三内科

氏名松田正之

TEL 0263-37-2673

FAX 0263-37-3427

E-mail matsuda@hsp.md.shinshu-u.ac.jp 


\section{Abstract}

Objective: Immunoglobulin-related free light chains (FLCs) in serum have recently become quantitatively detectable using the nephelometric assay in plasma cell disorders, including multiple myeloma and AL amyloidosis. To investigate whether FLCs are useful as a diagnostic and therapeutic marker in Japanese patients with primary systemic AL amyloidosis, we determined these values in serum before and after chemotherapy.

Patients and methods: The serum FLC analysis was carried out in 25 patients with primary systemic AL amyloidosis (mean age, 60.1 8.4 years). All of the patients were

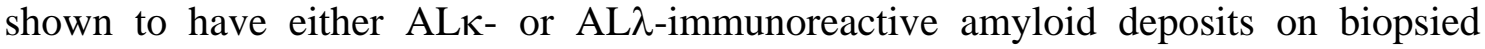
tissues. Thirteen patients were treated with VAD (vincristine, doxorubicin and dexamethasone) alone ( $\mathrm{n}=6$ ) or VAD and subsequent high-dose melphalan followed by autologous stem cell support $(\mathrm{n}=7)$, and serum FLCs were serially determined before and after the chemotherapy.

Results: Before chemotherapy the amyloidogenic FLC was elevated in serum with or without abnormal $\kappa / \lambda$ ratios in 24 patients, including 5 with undetectable M-protein in both serum and urine on immunofixation. After chemotherapy the amyloidogenic FLC in serum was significantly decreased irrespective of high-dose melphalan ( $\mathrm{p}<0.05$ ), and all the patients with normalized $\kappa / \lambda$ ratios showed a good prognosis.

Conclusions: With respect to sensitivity and quantification serum FLCs will be a key marker for diagnosis and therapeutic effects in primary systemic AL amyloidosis. The prognosis of patients with this disease may be improved if the $\kappa / \lambda$ ratio in serum can be normalized by intensive chemotherapy.

Key words: AL amyloidosis, autologous peripheral blood stem cell transplantation, free light chain, high-dose melphalan, immunofixation, M-protein, VAD 


\section{Introduction}

Primary systemic AL amyloidosis is characterized by progressive dysfunction of multiple vital organs due to extracellular deposition of amyloid. In this disease amyloid is derived from immunoglobulin light chains produced by plasma cell dyscrasia; thus, the precursor protein designated as M-protein is usually present in serum and/or urine (1, 2). Although protein electrophoresis and immunofixation have been used for detecting M-protein in primary systemic AL amyloidosis, these methods are not entirely satisfactory with regard to sensitivity and quantification. Recently, a sensitive nephelometric assay for free light chains (FLCs) has become usable in plasma cell disorders, including multiple myeloma and AL amyloidosis, and has shown reliable results, particularly for evaluating the therapeutic effects of chemotherapy (3-7). This assay is specific for $\kappa$ - and $\lambda$-type FLCs, and does not recognize light chains bound to the immunoglobulin heavy chain (4). In this study we determined serum levels of FLCs before and after chemotherapy in Japanese patients with primary systemic AL amyloidosis, and investigated whether these values were useful as a diagnostic and therapeutic marker. Serum levels of the amyloidogenic FLC were increased in all except one of these patients, and were significantly decreased after chemotherapy. We postulate that serum FLCs can be employed as an optional marker for the diagnosis and treatment of primary systemic AL amyloidosis instead of protein electrophoresis and immunofixation because of high sensitivity and easy quantification.

\section{Patients and methods}

\section{$\underline{\text { Patients }}$}

We studied 25 Japanese patients with primary systemic AL amyloidosis who were referred to our hospital between April 2000 and May 2004 (15 men and 10 women; age range, 44 to 78 years; mean, $60.1 \pm 8.4$ years). None of the patients had received any medical treatment for plasma cell dyscrasia prior to being referred to us. The diagnosis 
of this type of amyloidosis was based on two criteria: apparent deposition of amyloid with evidence of AL type in more than 2 biopsy sites, and no associated disorder possibly underlying the amyloidosis. To detect amyloid deposition, conventional alkaline Congo red staining was performed in several biopsied tissues such as the kidney and gastroduodenal mucosa, and in at least one of these specimens immunohistochemical staining was carried out using different antibodies to ALא, AL $\lambda$, amyloid $A$, transthyretin and $\beta 2$ microglobulin in order to confirm it as AL type (8). Multiple myeloma was excluded by serum M-protein lower than $3.0 \mathrm{~g} / \mathrm{dl}$, plasma cells less than $10 \%$ of the total number of nucleated cells in the bone marrow and no related organ or tissue impairment according to the diagnostic criteria proposed by the International Myeloma Working Group (9). The percentage of plasma cells in the bone marrow was examined on smear specimens treated with Wright-Giemsa staining. To identify M-protein in serum and urine, immunofixation was performed in all patients using a commercially available kit (Titan Gel IFE kit, Helena Laboratories, Saitama, Japan), and other information was obtained from their medical records. At study entry the extent of amyloid-related organ involvement was evaluated in each patient according to clinical criteria proposed by Comenzo et al (10).

\section{Treatment}

We administered VAD (vincristine, doxorubicin and dexamethasone) and subsequent high-dose melphalan with auto-PBSCT to 7 patients and VAD alone to 6, following our eligibility criteria (11). Details of the intensive chemotherapy are described elsewhere $(11,12)$. Briefly, stem cells were collected using etoposide and granulocyte-colony stimulating factor after 2 courses of VAD as an induction treatment, and high-dose melphalan was administered intravenously at $140 \mathrm{mg} / \mathrm{m}^{2}$ total dose over 2 days. Hematopoietic stem cells were infused 48 hours later. The VAD regimen is as follows: vincristine $0.4 \mathrm{mg} /$ day and doxorubicin $9 \mathrm{mg} / \mathrm{m}^{2} /$ day by continuous intravenous infusion on days 1-4, and dexamethasone $40 \mathrm{mg} /$ day by intravenous 
infusion on days 1-4, 9-12 and 17-20. In the patients treated with VAD alone this regimen was repeatedly performed until M-protein disappeared from both serum and urine on immunofixation, when their general status was satisfactory without any serious involvement of visceral organs, particularly signs of congestive heart failure.

\section{$\underline{\text { Serum FLC assay }}$}

Blood was taken from all subjects before and after the chemotherapy, and serum was cryopreserved at $-80^{\circ} \mathrm{C}$ with sodium azide as preservative until the assay. FLCs were measured in stored serum samples using a commercially available kit based on a latex-enhanced immunoassay (The Binding Site, Birmingham, UK) on a Behring BN II nephelometric analyzer (Dade Behring, Deerfield, IL, USA). The assay utilizes antibodies against FLC epitopes that are hidden in whole immunoglobulin molecule. In each subject both $\kappa$ - and $\lambda$-type FLCs were separately determined, and the $\kappa / \lambda$ ratio was calculated. Normal values in healthy subjects are as follows: $3.3-19.4 \mathrm{mg} / \mathrm{l}$ for $\kappa, 5.7-26.3 \mathrm{mg} / \mathrm{l}$ for $\lambda, 0.26-1.65$ for the $\kappa / \lambda$ ratio.

\section{$\underline{\text { Statistics }}$}

To detect statistically significant changes after chemotherapy, Wilcoxon's signed rank test was employed for serum levels of the amyloidogenic FLC. The results represent the mean \pm standard error where applicable, and a p-value less than 0.05 was considered to be statistically significant. Commercially available statistics software was used for data analysis (StatView for Macintosh, Abacus Concepts, Berkeley, CA, USA).

\section{Results}

Clinical profiles of the patients are summarized in Table 1. Amyloid in tissues was

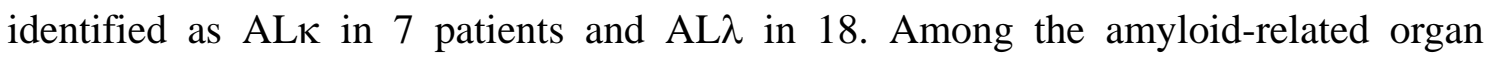
disorders proteinuria was the most frequent, and an abnormality in electro- and/or echocardiography was second. On immunofixation M-protein in serum was confirmed as IgG $\lambda$ in 7 patients (cases 1,8 to 10 and 14 to 16), IgA $\lambda$ in 3 (cases 6,7 and 19), 


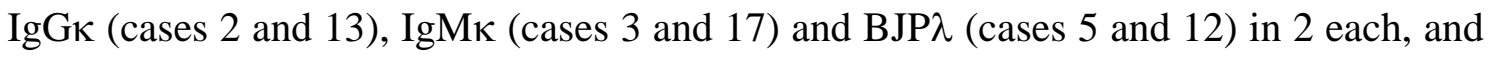
IgM $\kappa$ and $\lambda$ in 1 (case 18). Three patients (cases 4, 11 and 20) showed BJP $\lambda$ in urine but not in serum. In the remaining 5 patients M-protein was undetectable in both serum and urine. All the patients showed an increase in either $\kappa$ - or $\lambda$-type FLCs except for case 6. The FLCs that were predominantly increased in serum completely coincided with the types of immunoglobulin light chain shown by immunofixation and/or immunohistochemical analysis of amyloid deposits. Immunofixation demonstrated no M-protein in serum in cases 4, 11 and 20 to 25 while the serum FLC analysis showed positive results in all 8 patients.

Results of immunofixation and the FLC analysis 3 months after the chemotherapy are demonstrated in Table 2. Two courses of VAD and high-dose melphalan with auto-PBSCT were given in cases 1 to 7 , and cases 8 to 13 were treated with VAD alone. Serum levels of the amyloidogenic FLC were reduced by more than $50 \%$ in all the patients except for one (case 11). In the group treated with VAD and high-dose melphalan, 5 patients showed disappearance of M-protein in both serum and urine on immunofixation after the treatment, although slightly elevated levels of amyloidogenic FLC were seen in cases 2 and 4 . In case 5 M-protein was still positive in both serum and urine on immunofixation, and the amyloidogenic FLC stayed at a high level. Case 6 showed normal levels of the amyloidogenic FLC after the treatment, but M-protein was detectable in serum on immunofixation. In the group treated with VAD alone, 3 patients (cases 8, 9 and 13) showed disappearance of M-protein in both serum and urine on immunofixation after 2 courses of chemotherapy. Amyloidogenic FLC in serum also decreased to almost normal levels, and VAD was stopped. Although in cases 10 and 11 M-protein was still positive on immunofixation with elevated levels of amyloidogenic FLC after 2 courses of VAD, this therapy could not be continued because of their bad general status with worsening of organ dysfunction. Since in case 12 the amyloidogenic FLC still showed a high value after 2 courses of VAD despite disappearance of 
M-protein on immunofixation, a third cycle of this therapy was additionally performed after an interval of 3 months. After that the amyloidogenic FLC decreased to the normal level.

Serial studies of the amyloidogenic FLC in serum are demonstrated in Figure 1. There was a significant decrease in serum levels of the amyloidogenic FLC 1 to 2 months after VAD (162.4 $\pm 96.8 \mathrm{mg} / \mathrm{l}, \mathrm{p}<0.05)$ and 3 months after high-dose melphalan (78.6 $\pm 58.6 \mathrm{mg} / \mathrm{l}, \mathrm{p}<0.05)$ compared with before $(299.0 \pm 184.4 \mathrm{mg} / \mathrm{l})$ in the patients treated with 2 courses of VAD and high-dose melphalan followed by auto-PBSCT. None of the patients showed any reincrease in serum levels of the amyloidogenic FLC 9, 15 and 27 months after high-dose melphalan. In the patients treated with VAD alone, serum levels of the amyloidogenic FLC were significantly decreased 3 months after the chemotherapy (104.9 $\pm 46.8 \mathrm{mg} / \mathrm{l}, \mathrm{p}<0.05)$ compared with before $(459.6 \pm 183.9 \mathrm{mg} / \mathrm{l})$. None of the patients showed any reincrease in serum levels of the amyloidogenic FLC 9 months after the chemotherapy.

The patients were divided into 2 groups according to normalization of the $\kappa / \lambda$ ratio 3 months after the chemotherapy. The $\kappa / \lambda$ ratio was normalized in 8 patients, and of these 7 showed obvious improvement in visceral organ involvement: multiple gastroduodenal hemorrhagic lesions ameliorated in case 2, and cases 1, 3, 4 and 7 to 9 showed improvement of nephrotic syndrome with an increase in serum levels of albumin and a decrease in daily protein excretion in urine. Normalization of the $\kappa / \lambda$ ratio was not seen in the remaining 5 patients, and of these 3 showed worsening of clinical symptoms: cases 5 and 10 developed congestive heart failure, and amyloidosis-related renal dysfunction deteriorated and resulted in permanent hemodialysis in case 11. All 3 patients died of amyloidosis-related organ failure. Kaplan-Meier's survival curves of the 2 groups are demonstrated in Figure 2. The fifty-percent survival period was 232.0 460.7 days for the group with abnormal $\kappa / \lambda$ ratios after chemotherapy $(n=5)$, while it could not be calculated for that of the 
normal $\kappa / \lambda$ ratios $(n=8)$ because all the patients are still alive. The number of patients alive 300 days after commencement of therapy was 8 in the group with normalized $\kappa / \lambda$ ratios and 2 in the group with abnormal $\kappa / \lambda$ ratios.

\section{Discussion}

Immunohistochemical characterization of amyloid deposits is most essential for the diagnosis of AL amyloidosis, but M-protein in serum and/or urine is also important as evidence of plasma cell dyscrasia, which is the pathological basis particularly for the systemic type of this disease. To detect M-protein in serum and/or urine, protein electrophoresis and immunofixation have been employed for many years. In primary systemic AL amyloidosis, however, these conventional techniques are often inadequate for evaluating M-protein because the concentration is too low in many patients, in contrast to multiple myeloma (2). In this study we employed the FLC analysis which has appeared in the last 5 years in order to detect M-protein in serum (3-7), as it is much more sensitive in detecting M-protein than conventional methods. Serum protein electrophoresis and immunofixation typically have detection limits of 500 to $2000 \mathrm{mg} / \mathrm{l}$ and 150 to $500 \mathrm{mg} / \mathrm{l}$, respectively, whereas the serum FLC analysis usually shows a sensitivity of less than $5 \mathrm{mg} / \mathrm{l}$ (7). According to a recent report, the FLC analysis could identify serum monoclonal protein in more than $98 \%$ of patients with AL amyloidosis (7). Also in the present study, serum levels of the amyloidogenic FLC were increased with or without abnormal $\kappa / \lambda$ ratios in 24 patients (96\%), including 5 with M-protein undetectable in both serum and urine on immunofixation. A relative excess of $\kappa$ - or $\lambda$-type FLCs correctly identified the amyloidogenic FLC class in each patient. These results suggest that the FLC analysis is superior to immunofixation with respect to sensitivity for detecting M-protein in serum, and might be able to facilitate the diagnosis of primary systemic AL amyloidosis. When monoclonal light chains are present in serum mostly unbound to heavy chains, the amyloidogenic FLC is likely to show 
considerably high levels despite negative results on immunofixation, as in cases 23 and 24.

To sensitively evaluate therapeutic effects of the chemotherapy, quantification of M-protein is necessary. In this respect, the FLC analysis is obviously superior to protein electrophoresis and immunofixation, although the antibodies in this assay cannot distinguish monoclonal FLCs from low-level polyclonal ones that exist also in the healthy population. In the present study, 5 of 13 patients showed complete normalization in all indices of the serum FLC analysis after the chemotherapy in conjunction with disappearance of M-protein on immunofixation, while in 3 patients both examinations remained positive. In 4 patients serum levels of the amyloidogenic FLC were decreased after the chemotherapy but still positive, although M-protein became undetectable in both serum and urine on immunofixation. These results suggest that the FLC analysis coincided well with immunofixation, but the former might be more useful than the latter in the follow-up of the patients after chemotherapy because of its ability to detect minute changes in M-protein. In one patient (case 6), however, the FLC analysis showed neither elevated levels of the amyloidogenic FLC nor abnormal $\kappa / \lambda$ ratios throughout the clinical course despite positive M-protein on immunofixation. Since the whole immunoglobulin is usually undetectable in the FLC analysis (4), monoclonal light chains may have been present in serum bound to heavy chains in this patient. In such a case immunofixation would be better than the FLC analysis in evaluating the therapeutic effects of the chemotherapy.

In general, serum levels of FLCs are dependent on renal function, and sometimes increase in association with reduced glomerular filtration $(7,13)$. In the present study, several patients showed elevated levels of the non-amyloidogenic FLC in serum, which were ascribable to amyloid-induced renal dysfunction as well as increased production of polyclonal immunoglobulin. To precisely evaluate production of M-protein in primary systemic AL amyloidosis, which frequently involves the kidneys from the early phase of 
illness, the $\kappa / \lambda$ ratio is probably a better marker than the concentration of FLCs because it is unaffected by renal function $(7,13)$. With regard to survival after the chemotherapy all the patients with normalized $\kappa / \lambda$ ratios are still alive in the present study. This result suggests that the intensive chemotherapy should be performed in primary systemic AL amyloidosis for normalization of the $\kappa / \lambda$ ratio in serum in order to improve the prognosis. According to a recent report, the survival rate was significantly better if the amyloidogenic FLC fell by more than half after the chemotherapy (7). In the present study, however, the amyloidogenic FLC in serum failed to fall more than half only in one patient after the chemotharapy, and it could not be assessed whether the magnitude of a decrease in the amyloidogenic FLC was significantly related to the survival rate or not.

In conclusion, serum levels of the amyloidogenic FLC can quantitatively and reliably be detected in serum using the nephelometric assay, and are useful as a marker for the diagnosis of primary systemic AL amyloidosis and evaluation of the therapeutic effects of the chemotherapy. The $\kappa / \lambda$ ratio after the chemotherapy might be able to more sensitively predict the prognosis of the patients than serum levels of the amyloidogenic FLC, particularly in patients with amyloid-induced renal dysfunction.

\section{Acknowledgement}

The authors are grateful to Drs. Y. Hoshii and T. Ishihara, Department of Pathology, Yamaguchi University School of Medicine, for their help with immunohistochemical staining of the biopsy specimens. This work was supported by a grant from the Intractable Disease Division, the Ministry of Health and Welfare, Amyloidosis Research Committee, Japan. 


\section{References}

1) Buxbaum J. The amyloidoses. in: Rheumatology. 2nd ed. Klippel JH, Dieppe PA, Eds. Mosby, London, 1998: 8.27.1-10.

2) Kyle RA, Gertz MA. Primary systemic amyloidosis. Clinical and laboratory features in 474 cases. Semin Hematol 32: 45-59, 1995.

3) Drayson M, Tang LX, Drew R, Mead GP, Carr-Smith H, Bradwell AR. Serum free light-chain measurements for identifying and monitoring patients with nonsecretory multiple myeloma. Blood 97: 2900-2902, 2001.

4) Bradwell AR, Carr-Smith HD, Mead GP, et al. Highly sensitive, automated immunoassay for immunoglobulin free light chains in serum and urine. Clin Chem 47: 673-680, 2001.

5) Katzmann JA, Clark RJ, Abraham RS, et al. Serum reference intervals and diagnostic ranges for free $\kappa$ and free $\lambda$ immunoglobulin light chains: relative sensitivity for detection of monoclonal light chains. Clin Chem 48: 1437-1444, 2002.

6) Abraham RS, Katzmann JA, Clark RJ, Bradwell AR, Kyle RA, Gertz MA. Quantitative analysis of serum free light chains. A new marker for the diagnostic evaluation of primary systemic amyloidosis. Am J Clin Pathol 119: 274-278, 2003.

7) Lachmann HJ, Gallimore R, Gillmore JD, et al. Outcome in systemic AL amyloidosis in relation to changes in concentration of circulating free immunoglobulin light chains following chemotherapy. Br J Haematol 122: 78-84, 2003.

8) Hoshii Y, Setoguchi M, Iwata T, et al. Useful polyclonal antibodies against synthetic peptides corresponding to immunoglobulin light chain constant region for immunohistochemical detection of immunoglobulin light chain amyloidosis. Pathol Int 51: 264-270, 2001. 
9) The International Myeloma Working Group. Criteria for the classification of monoclonal gammopathies, multiple myeloma, and related disorders: a report of the International Myeloma Working Group. Br J Haematol 121: 749-757, 2003.

10) Comenzo RL, Vosburgh E, Falk RH, et al. Dose-intensive melphalan with blood stem-cell support for the treatment of AL amyloidosis: survival and responses in 25 patients. Blood 91: 3662-3670, 1998.

11) Gono T, Matsuda M, Shimojima $Y$, et al. VAD with or without subsequent high-dose melphalan followed by autologous stem cell support in AL amyloidosis: Japanese criteria for patient selection and our experiences. Amyloid: J Protein Folding Disord 11: 245-256, 2004.

12) Gono T, Matsuda $M$, Dohi $N$, et al. Nephrotic syndrome due to primary AL amyloidosis, successfully treated with VAD and subsequent high-dose melphalan followed by autologous peripheral blood stem cell transplantation. Intern Med 42: 72-77, 2003.

13) Clark RJ, Katzmann JA, Abraham RS, Lymph JF, Kyle RA, Bradwell AR. Detection of monoclonal free light chains by nephelometry: normal ranges and relative sensitivity. Clin Chem 47: a27, 2001. 


\section{Figure legends}

Figure 1: In the patients treated with VAD and subsequent high-dose melphalan followed by autologous stem cell support (A), there were significant differences in serum levels of the amyloidogenic FLC between before and 1 to 2 months after VAD $(\mathrm{p}<0.05)$ or 3 months after high-dose melphalan $(\mathrm{p}<0.05)$. Also in the patients treated with VAD alone (A), serum levels of the amyloidogenic FLC were significantly decreased 3 months after the chemotherapy compared with before $(p<0.05)$. The number of patients examined is shown in parentheses. *FLCs were not determined 1 to 2 months after VAD in case 7. VAD: vincristine, doxorubicin and dexamethasone, FLC: free light chain.

Figure 2: Kaplan-Meier's survival curves of 2 patient groups classified according to normalization of the $\kappa / \lambda$ ratio 3 months after the chemotherapy. Solid line: patients with normalized $\kappa / \lambda$ ratios $(n=8)$, broken line: those with abnormal $\kappa / \lambda$ ratios $(n=5)$. Censored patients were ticked along each survival curve. 